\title{
What are the specifics of modern treatment of acute pneumonia?
}

\author{
Dr. Igor Klepikov ${ }^{1^{*}}$ \\ 1 Professor, Renton, Washington, USA \\ *Corresponding Author: Dr. Igor Klepikov, MD, Professor, Renton, Washington, USA Email: igor.klepikov@yahoo.com
}

Received: February 22, 2019; Published: March 28, 2019

If a letter or appeal begins with a question, the answer to it is usually in the final part of the message after preliminary explanations. However, in this situation, I will not intrigue readers and immediately give an answer to the question that I myself put. From my point of view, modern treatment of acute pneumonia (AP), which is widespread and generally accepted at the level of official medicine around the world, does not contain any specific methods or drugs intended only for this disease. Looking at the list of recommendations for the treatment of patients with AP, you will see a standard set of General Therapeutic agents and treatments that are used in various pathologies, different localization, clinical picture and mechanisms of development.

Focusing all attention on the suspected pathogen AP and its suppression, such recommendations completely ignore the unique features of the disease. The complex of therapeutic efforts is based on the representation of AP as an infectious process and is automatically borrowed from the experience of treatment of other diseases. The use of various procedures in AP did not pass the test of their influence on the dynamics of the process in the lungs. The assessment of the effectiveness or inefficiency of the various methods of assistance is based on impressions and circumstantial evidence rather than objective criteria. The habit and stability of such approaches to AP treatment have become commonplace, and no one notices the depravity of the situation. But this situation is the cause of dissatisfaction with the results of AP treatment and the observed trend of their deterioration. To understand the anatomy of the modern AP treatment process, it is necessary to look at the typical conditions of step-by-step care for such patients.

In the clinic, the family doctor examined the patient and diagnosed AP. Based on the information received, the doctor believes that treatment at home is quite acceptable in this situation. The means of treatment in this case are intended for the patient himself without the participation of medical personnel, and therefore, as a rule, medical prescriptions should be easily fulfilled. The main emphasis in these recipes, except for a number of symptomatic agents, is on antibiotics. The doctor does not have additional and accurate information about the causative agent of the inflammatory process in the lungs, therefore, determines the choice of an antibiotic based on the prevailing ideas about the etiology of the disease and his own experience. In such situations, bacterial infection is assumed and the likelihood of another etiology (primarily viral) usually does not affect therapeutic purposes.

Continuation of such a typical history can have two the most possible outcome. In the first case, the initiated treatment gives an undoubted effect and the patient recovers, often receiving recommendations for the extension of the course of antibiotic therapy to consolidate the achievements. With this outcome, doctors usually consider a positive result as their own victory, considering the empirical choice of an antibiotic successful, but not knowing the true etiology of the disease. However, the real circumstances more suggest that the patient won the fight. As you know, antibiotics are designed only to suppress microbes and have no other therapeutic effects. Inflammatory tissue changes and functional disorders in the body are completely dependent on the protective and adaptive capabilities of the patient. In addition, the body's reaction to inflammation was not characterized by expression and rapid dynamics of changes. In such a situation, the suppression of the activity of the accompanying microflora is enough for the body to cope with the problem on its own. In this situation, on the contrary, it is necessary to sensibly assess the real role of the doctor in the elimination of the disease.

A detailed examination of the patient and the correct diagnosis reflect the high competence and professionalism of the doctor. However, as soon as it comes to the choice of treatment, decisions and actions of the doctor become frankly simple and primitive. Writing a prescription for an antibiotic, the doctor does not think that today he has already prescribed this medicine to patients with completely different forms of inflammatory processes-with otitis media, with acute tonsillitis, with inflammation of soft tissues, with pyelonephritis, etc. In fact, the doctor who serves patients with inflammatory diseases in the clinic, is engaged in the distribution of "one drug" between different groups of patients, and its function in this section is more like the work of a pharmacist or even a distributor of a pharmaceutical company than a doctor. In addition, such a narrow focus and limited choice of drugs in the treatment of AP is an indirect evidence of the perception of antimicrobials as a universal remedy for many diseases. This misconception has not yet been seriously revised, as the principle of treating only "antibiotics alone" still helps many patients, despite the increase in the number of resistant strains, but only through the development of new drugs. This traditional focus on antibiotics now underlies daily practice and is inextricably linked to the dominant perception of AP as an infectious process. Surprisingly, the stability of these therapeutic principles remains unchanged, despite the tendency to reduce their effectiveness 
One of the variants of development of events after AP diagnostics was considered. This option is the most profitable, almost ideal, but it does not give a clear idea of the essence of the accumulated problems. The fog of illusions begins to disappear when the expected effect of the initiated treatment is not received and there is a need for hospitalization of the patient. In other words, the hope for antibiotics as a panacea is melting and requires strengthening and supplementing the treatment complex. A similar situation is observed in the aggressive onset of the disease, in this case, the patient is sent to the hospital immediately after the diagnosis of AP. In such situations, now no one expects a lightning effect, as in the first years of antibiotic therapy and therefore from the moment of hospitalization, patients with AP receive comprehensive care as suffering from an infectious process.

Thus, a look at the nature of the disease determines the treatment strategy. Since the fight against infection is at stake, the direction has not changed. Antibiotic therapy remains the leading part of the treatment program, which is complemented by The General therapy of the observed syndromes. As a result, specialized medical care in AP not only does not have its own specifics, but is almost identical to the treatment of completely different diseases, for example, such as diarrhea. In the latter comparison, the difference may be in the choice of antibiotics. In addition, in case of intestinal infections, material for bacteriological examination can be obtained directly from the affected area, while with AP, such a possibility appears only with the development of purulent complications.

Despite the fact that the patient with AP in the hospital is subjected to various examinations and constant monitoring, the choice of an antibacterial drug for it is determined empirically [13]. In most patients, the probability of taking the material directly from the area of inflammation is impossible, therefore, as a rule, the microflora of the nasopharynx and oropharynx is investigated [4-6]. The results of such studies may not be an accurate indicator of AP etiology. Firstly, often the microflora of the upper respiratory tract in healthy people may contain more virulent strains, than in patients with AP [7]. However, the presence of opportunistic aggressive bacteria in the body does not necessarily mean the development of inflammation. Secondly, today there are more than 100 microorganisms that can cause inflammation in the lungs [8], and not only a small group of bacteria that are constantly featured in literary publications. Finally, what to do with patients whose AP is caused by pathogens such as viruses, fungi, etc. For example, according to modern ideas, about a third of AP patients in the world have a viral etiology of the disease $[1,9]$. Thus, there is a very strange situation in the treatment of AP. On the one hand, the fear of microbial pathogen AP is the basis of all therapeutic measures that are currently used in this disease, and determines the entire strategy of care. On the other hand, in the vast majority of these patients, the main culprit of this situation has no objective and undoubted ways of verification and in fact remains unknown.

The first procedure that almost every patient with AP receives additionally from the moment of hospitalization is intravenous infusion of solutions. If patients with intestinal infection intravenous fluid is justified because of its large losses through diarrhea and vomiting, such assistance in AP is purely declarative and has a very illusory rationale. The more severe the condition of patients with $\mathrm{AP}$, the more intense intravenous infusion [1,2,10-15]. Most often, the need for the use of infusions in patients with AP is explained by the loss of fluid by perspiration. At the same time, anybody and never conducted special studies and provided objective evidence of large losses in AP by perspiration never argued the urgent need and usefulness of such assistance.

In addition, the need for drip infusions in AP is explained by the presence of so-called intoxication in these patients. This term usually refers to the definition of the General condition of patients, has no clear criteria and is widely used in the description of various diseases. Further dissemination of the term "intoxication", which is a very amorphous definition, will create a false perception and play a negative role in understanding the mechanisms of the disease. As for the AP, the last point is of fundamental importance. The essence of this replica is as follows. It is time to realistically and thoughtfully assess the main differences between AP and other inflammatory processes. This disease is the only acute nonspecific inflammatory process that occurs in the area of the small circle of blood circulation. All other nosologies of this category have localization of inflammation in the vascular system of the great circle of blood circulation. This fact is undoubted and is not subject to discussion, but it determines the uniqueness of the mechanisms of AP development.

The leading role of pulmonary vessels in the automatic regulation of the balance between small and large circles of blood circulation is well known. For synchronous operation of the right and left parts of the heart, it is necessary that the pressure in the vessels of the small circle is several times lower than in the large circle. The beginning of inflammation in the lung tissue creates a zone of reflex effect, which increases the tonus of the pulmonary vessels. Reflex spasm of vessels reduces the capacity of the small circle, to discharge which the body must reduce the pressure in the large circle and additionally have time to deposit part of the circulating blood. In this situation, the reactivity of the body is important, which determines the speed of development of the inflammatory reaction and its consequences. The rapid development of the noted mechanisms of restructuring the entire circulatory system leaves no time and opportunities for adaptation to new conditions. Consequently, in most severe cases of AP there is a typical pattern of shock, only this version of shock is not septic, as it is currently interpreted. This variant of shock was previously identified by us as pulmonal shock, which in contrast to the actual septic shock has fundamental differences in the mechanism of its occurrence.

The described part of the pathogenesis of AP is a specific characteristic of this disease, which has an absolute difference from the general disorders in the body in inflammatory processes of other localization. This mechanism is not only a consequence of biological stereotypes and axioms, but also received additional confirmation in special studies and subsequent clinical trials [16-28].

To the above, we should add the fact that the modern interpretation of shock in AP as septic has no objective confirmation in the bacteriological study of blood in the vast number of patients. Thus, shock in AP in many patients has no microbiological characteristics, and among all hospitalized with the severe disease, a positive blood culture was registered only in $10-12 \%$, including in cases of bacteremia and sepsis $[1,2,13]$. These statistics of recent years are an additional contradiction of the septic nature of shock in AP. Automatic transfer of the General principles of shock therapy to the group of patients with AP was based on insufficient representation of the disease pathogenesis and was not accompanied by objective testing of their influence on the current processes. Evaluation of the role 
of infusion therapy in the dynamics of AP from new positions can give a completely different explanation for failures in the treatment of this group of patients. Additional infusion load on the vessels of the small circle of blood circulation gives a completely opposite effect instead of the expected. When the body ceases to resist this additional aggression, it is the turn of vasopressors and hormones $[1,11,15,29]$.

The final results of AP treatment for a long time remain a matter of concern for specialists who see the causes of all failures in the provision of medical care to these patients only through the prism of the microbial factor. The persistent focus of views on only one of the signs of the disease leaves without attention and proper assessment of its leading mechanisms that determine the entire specificity of AP. These narrow views limit the principles of modern medical care for patients with AP, which deprives the medical complex of the necessary originality. In addition, the automatic transfer of General therapeutic principles of care to patients with AP without prior informed testing can sometimes play the role of an additional incentive for pathological transformation.

The above considerations are not only derived from the functional role of the lungs in the body and biological stereotypes of inflammatory processes, but also confirmed by additional studies and clinical trials. All these facts give the reader a reason to critically evaluate the modern concepts of AP and the existing principles of its treatment.

\section{REFERENCES}

1. Morgan AJ, AJ Glossop BM. Severe community-acquired pneumonia. BJA Education. 2016;16(5):167-72.

2. Sethi S. Merck Manual Professional Edition / Pulmonary Disorders / Aspiration Pneumonitis and Pneumonia. Last full review/revision December 2014.

3. Prina E, Ranzani OT, Torres A. Community-acquired pneumonia The Lancet. 2015;386(9998):1097-108.

4. https://www.cdc.gov/media/releases/2015/p0225-pneumoniahospitalizations.html

5. Jain S, Williams DJ, Arnold SR, Ampofo K, Bramley AM, Reed C et al. Community-acquired pneumonia requiring hospitalization among US children. New England Journal of Medicine. 2015;372(9):835-45.

6. Mani CS, Murray DL. Acute Pneumonia and Its Complications. Part II: Clinical Syndromes and Cardinal Features of Infectious Diseases: Approach to Diagnosis and Initial Management. SECTION D: Lower Respiratory Tract Infections. In the book: S.S. Long, L.K. Pickering, C. G. Prober. Principles and Practice of pediatric infectious diseases. Edinburgh, New York: Elsevier Churchill Livingstone, (2012): 235-245.

7. Davis CP. Normal flora. InMedical Microbiology. 4th edition 1996. University of Texas Medical Branch at Galveston.

8. https://en.wikipedia.org/wiki/Pneumonia

9. Ruuskanen O, Lahti E, Jennings LC, Murdoch DR. Viral pneumonia. The Lancet. 2011;377(9773):1264-75.

10. Ferrer M, Travierso C, Cilloniz C, Gabarrus A, Ranzani OT, Polverino E, Liapikou A, Blasi F, Torres A. Severe communityacquired pneumonia: Characteristics and prognostic factors in ventilated and non-ventilated patients. Plos one. 2018;13(1):e0191721.

11. Liapikou A, Rosales-Mayor E, Torres A. The management of severe community acquired pneumonia in the intensive care unit. Expert review of respiratory medicine. 2014;8(3):293-303.

12. Kim JW, Kim JJ, Yang HJ, Lim YS, Cho JS, Hwang IC et al. The Prognostic Factors of Pneumonia with Septic Shock in Patients Presenting to the Emergency Department. Korean Journal of Critical Care Medicine. 2015;30(4):258-64.

13. Waterer GW, Quasney MW, Cantor RM, Wunderink RG. Septic shock and respiratory failure in community-acquired pneumonia have different TNF polymorphism associations. American journal of respiratory and critical care medicine. 2001;163(7):1599-604.
14. Lim TK, Chew MY. Management of severe community acquired pneumonia in the emergency department. Journal of Emergency and Critical Care Medicine. 2018;2(1):2.

15. Angus DC, Barnato AE, Bell D, Bellomo R, Chong CR, Coats TJ et al. A systematic review and meta-analysis of early goal-directed therapy for septic shock: the ARISE, ProCESS and ProMISe Investigators. Intensive care medicine. 2015;41(9):1549-60.

16. Klepikov I. Acute pneumonia and its purulent and destructive complications in children in the midst of a major industrial centre of Western Siberia. Diss. Dissertation for the degree of doctor of medical sciences. Leningrad, 1989.

17. Klepikov I, Rikov V. A method for modeling parapneumonic pleurisy. Author's certificate for invention. SU, No 1631574, A1, 1(1990).

18. Klepikov I. Acute pneumonia: a new look at the old problem. LAP LAMBERT Academic Publishing; 2017.

19. Klepikov I. The Meaning of Pulmonary Reflexes in the Pathogenesis of Acute Pneumonia. Intern Med. 2017;7(232):2.

20. Klepikov I. The Role of Cardiovascular Disorders in the Pathogenesis of Acute Pneumonia. J Cardiol \& Cardiovasc Ther. 2017;4(1):555628

21. Klepikov I. Illusory Nature of the Etiology of Acute Pneumonia. Acute Pneumonia is More Cardiovascular than Respiratory Disaster. J Emerg Med Care. 2018;1(1):105.

22. Klepikov I. First Aid for Aggressive Forms of Acute Pneumonia. EC Pulmonology and Respiratory Medicine. 2018;7(2):34-37.

23. Klepikov I. Cupping Therapy as a means of First Aid in Acute Pneumonia. J Clin Case Stu. 2018;3(2):1-3.

24. Klepikov I. The Effect of Intravenous Infusion on the Dynamics of Acute Pneumonia. EC Pulmonology and Respiratory Medi-cine. 2017:4:15-20.

25. Klepikov I. Dependence of the results of treatment of acute pneumonia on the doctrine of the disease. J Clin Intensive Care Med. 2018;3:10-12.

26. Klepikov I. Shock in Acute Pneumonia and its Mechanism.EC Emergency Medicine and Critical Care. 2018;2(2):52-53.

27. Klepikov I. Angioarchitectonics of Acute Pneumonia. J Clin Intensive Care Med. 2019;4:018-022.

28. Klepikov I. Acute Pneumonia: Biological Rules and Laws require Attention and Respect. Journal of Respiratory Diseases. 2019;1(1):25-29.

29. Dellinger RP, Levy MM, Rhodes A, Annane D, Gerlach H, Opal SM, Sevransky JE, Sprung CL, Douglas IS, Jaeschke R, Osborn TM. Surviving Sepsis Campaign: international guidelines for management of severe sepsis and septic shock, 2012. Intensive care medicine. 2013;39(2):165-228. 